\section{Niveles de vitamina $D$ en médicos: ¿son los anestesiólogos el único grupo de riesgo?}

\author{
DAGOBERTO OJEDA ${ }^{1}$, MARIANA CABEZÓN ${ }^{\mathrm{a}}$, \\ MARIELA AGURTO ${ }^{1}$, SOFÍA OVIEDO ${ }^{2}$, CARLOS VEGA $^{\mathrm{b}}$, \\ XIMENA DAZA $^{\mathrm{b}}, \mathrm{BEATRIZ} \mathrm{DEMETRIO}^{\mathrm{c}}$, \\ CECILIA CARRASCO $^{\mathrm{c}}$, PATRICIA CISTERNAS ${ }^{1}$
}

\section{Vitamin D levels among anesthesiologists and other physicians}

Background: Sun exposure is the main source of 25-hydroxy-vitamin D. Since anesthesiologists work inside operating rooms, they are identified as a deficiency risk group. As medical activity in general occurs indoors, added to the work excess and sedentary lifestyle, physicians in general have low sun exposure. Aim: To investigate the determinants of vitamin D levels in physicians. Material and Methods: Anesthesiologists and physicians not working in operating rooms were included. A survey that comprised working hours, diet, skin color, sunscreen use and outdoor activities was also applied. Measurements of vitamin $D$ and parathormone levels in blood were performed. Results: We analyzed samples from 81 volunteers. Median vitamin D values of the whole sample were in the range of insufficiency (25.3 [interquartile range 12.4] $\mathrm{ng} / \mathrm{ml}$ ). Multiple linear regression analysis detected no differences between anesthesiologists and non-anesthesiologists. A higher body mass index was a risk factor for vitamin $D$ deficiency, $(p=0.025)$. The only protective factor was the intake of a vitamin $D$ supplement $(p<0.01)$. Conclusions: Anesthesiologists and other specialists were both at risk for vitamin D deficiency. Obesity was a risk factor and the use of a vitamin D supplement was the only protective factor.

(Rev Med Chile 2019; 147: 1415-1422)

Key words: Anesthesiologists; Physicians; Vitamin D Deficiency.

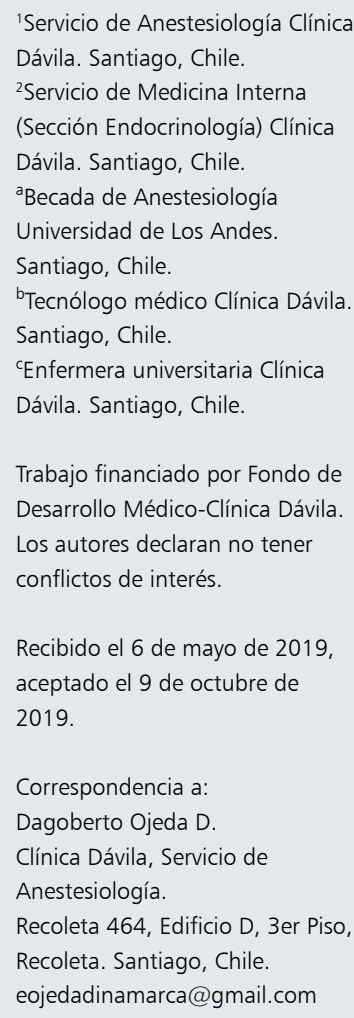

Recibido el 6 de mayo de 2019 aceptado el 9 de octubre de 2019.

Correspondencia a:

Dagoberto Ojeda D.

Clínica Dávila, Servicio de

Anestesiología.

Recoleta 464, Edificio D, 3er Piso,

Recoleta. Santiago, Chile.

eojedadinamarca@gmail.com

L a 25-hidroxi-vitamina D (vitamina D), participa en la homeostasis del calcio y juega un rol importante en el desarrollo y la preservación del aparato osteomuscular ${ }^{1,2}$. Las consecuencias de su déficit se han visto asociadas no solo a trastornos del aparato osteomuscular (debilidad muscular, fracturas, osteoporosis, caídas, etc.) sino también a demencia ${ }^{3}$, enfermedad de Alzheimer ${ }^{3}$, cáncer de próstata ${ }^{4}$, entre otras.

Siendo las fuentes dietéticas de vitamina D muy limitadas, el abastecimiento principal de esta vitamina radica en la síntesis cutánea inducida por la exposición al sol. Este mecanismo está limitado por factores como la latitud, altitud, hora del día, el uso de vestimenta, actividades deportivas al aire libre, color de la piel, edad, uso de bloqueador solar y la profesión que se desempeña ${ }^{5}$. Se ha identificado que las personas que trabajan de noche, profesionales de la salud y quienes trabajan en interiores tienen mayor riesgo de padecer déficit de vitamina $\mathrm{D}^{6}$.

Los anestesiólogos desarrollan su actividad al interior de los pabellones quirúrgicos, sin exposición a la luz solar, por lo cual no es raro que se les haya identificado como un grupo de riesgo para déficit de vitamina $\mathrm{D}^{5}$. Un artículo reciente de 
autores nacionales ${ }^{7}$, demostró la existencia de un déficit de vitamina $\mathrm{D}$ en anestesiólogos, especialmente en invierno. En base a estos antecedentes se decidió realizar un estudio para explorar los factores determinantes de los niveles de vitamina $\mathrm{D}$ en médicos de Clínica Dávila y definir si son los anestesiólogos el único grupo de riesgo en el área de la salud.

\section{Sujetos y Métodos}

Se realizó un estudio observacional del tipo corte transversal, para el cual se solicitó la aprobación del Comité Ético-Científico de Clínica Dávila. Se invitó a participar a los médicos de nuestra institución, tanto del servicio de anestesiología (incluyendo a los residentes) como a médicos que desempeñaran su labor fuera del pabellón (internistas, pediatras, neurólogos, intensivistas, urgenciólogos). Se solicitó el consentimiento informado de los participantes y se tomó la muestra de sangre correspondiente. Los resultados fueron notificados a los voluntarios, junto con una orientación acerca de la corrección de las deficiencias cuando correspondiera.

El tamaño muestral fue calculado en base a la revisión sistemática de Sowah ${ }^{6}$, que reportó un $46 \%$ de déficit de vitamina $\mathrm{D}$ en médicos clínicos y que dicha deficiencia era al menos $20 \%$ mayor en quienes trabajaban en pabellón, lo que resultaba en una muestra de 50 anestesiólogos, con un error tipo I de $5 \%$ y una potencia de $80 \%$. Por otro lado, para pesquisar una diferencia en los niveles de vitamina $\mathrm{D}$ entre anestesiólogos e internistas, usando los datos de Skephedinsdottir ${ }^{5}$, con un punto de diferencia sobre una media de $28,2 \mathrm{ng} /$ $\mathrm{ml}$, (reportado en anestesiólogos), se requerían 23 voluntarios por grupo (con error tipo I de 5\% y potencia de $90 \%$ ).

Se aplicó además una encuesta a cada participante, que incluía preguntas referidas a características demográficas, dietéticas, exposición al sol y la clasificación del color de la piel de acuerdo al foto-tipo de Fitzpatrick ${ }^{8}$. La recolección de las muestras de sangre se realizó antes de las 16:00 hrs, entre septiembre y diciembre del año 2018 principalmente.

Se midieron niveles de vitamina $\mathrm{D}$ y parathormona $(\mathrm{PTH})$ en sangre. Se extrajeron $6 \mathrm{cc}$ de sangre a los voluntarios, e inmediatamente las muestras fueron protegidas de la luz y conservadas en frío hasta ser analizadas. La determinación de la vitamina $\mathrm{D}$ se realizó en suero con ensayo de electro quimioluminiscencia y la de PTH en muestra de plasma con ácido etiléndiaminotetracético (EDTA) y con electro quimioluminiscencia. Los valores de laboratorio ${ }^{9}$ utilizados para clasificar a los participantes fueron: niveles suficientes de vitamina $\mathrm{D} \geq 30$, insuficientes $<30 \mathrm{y} \geq 20 \mathrm{y}$ deficientes $<20$. Se consideraron valores normales de $\mathrm{PTH}^{10}$ los comprendidos entre 10 y $65 \mathrm{pg} / \mathrm{ml}$.

Las variables cuantitativas fueron resumidas con promedio \pm desviación estándar (DS) o mediana [rango intercuartílico] y las variables categóricas con proporciones. Se consideraron significativos los valores $p$ menores a $5 \%$, con intervalos de confianza de $95 \%$. El análisis estadístico se realizó con regresión lineal.

Las variables que demostraron un valor $\mathrm{p}$ significativo en el análisis univariado fueron consideradas para un modelo multivariado. Los resultados fueron reportados según lo recomendado en el texto de Morgan ${ }^{11}$ et al. El programa estadístico utilizado fue STATA 13.

\section{Resultados}

Se obtuvieron muestras de 85 médicos y resultados efectivos en 81 de ellos, de los cuales 59 eran anestesiólogos.

En la Tabla 1 se aprecia que la muestra estudiada estaba compuesta principalmente por adultos jóvenes, con una proporción equilibrada según género, eutróficos y en su mayoría anestesiólogos. La coloración de la piel era predominantemente clara y blanca-oliva, (Fitzpatrick II y III) ${ }^{8}$, la cuarta parte de ellos utilizaba un suplemento de vitamina $\mathrm{D}$, casi la mitad se exponía al sol y la gran mayoría ingería pescado al menos una vez por semana.

Los niveles de vitamina $\mathrm{D}$ en los médicos estuvieron bajo lo normal $\left(25,3[ \pm 12,4] n g \cdot \mathrm{ml}^{-1}\right)$, $65 \%$ de la muestra estaba bajo la suficiencia y $27 \%$ se ubicaba dentro de la categoría de deficiencia (Figura 1).

Pese a que los valores de vitamina $\mathrm{D}$ fueron más altos en anestesiólogos $\left(25,3[ \pm 11,4] n g \cdot \mathrm{ml}^{-1}\right)$ que en no anestesiólogos $\left(23,2[ \pm 14,7] n g \cdot \mathrm{ml}^{-1}\right)$ no hubo diferencia significativa entre ellos.

La mayoría de los anestesiólogos se ubicaban en el rango de la insuficiencia y de lo normal. Los participantes no anestesiólogos se distribuían 
principalmente entre la deficiencia y la insuficiencia, pero esto, nuevamente, no constituyó diferencia significativa entre los 2 grupos (Figura 2).

Se recurrió a una transformación logarítmica de los niveles de vitamina $\mathrm{D}$ para ajustarlos a una distribución normal y así realizar un análisis con regresión lineal.

El resultado del análisis univariado se observa

\section{Tabla 1. Demografía y características de los sujetos de estudio}

\begin{tabular}{|lc|}
\hline Variable & Resultado \\
\hline Tamaño muestral $(\mathrm{n})$ & 85 \\
\hline Edad* (años) & $36(25-83)$ \\
Mujeres (\%) & 51 \\
Índice de masa corporal** $\left(\mathrm{kg} / \mathrm{m}^{2}\right)$ & $24,6 \pm 3,1$ \\
Anestesiólogos (\%) & 73 \\
$\quad$ Sí & 27 \\
No & $45(28-84)$ \\
Horas semanales de trabajo* & \\
Color de la piel según Escala de Fitzpatrick (\%) & 18 \\
1 & 32 \\
2 & 41 \\
3 & 9 \\
4 & 42 \\
Actividades al aire libre (\%) & 65 \\
Uso de bloqueador solar (\%) & 25 \\
Uso de suplemento vitamina D (\%) & 70,6 \\
\hline Ingesta de alimentos ricos en vitamina D (\%)
\end{tabular}

*Mediana (Mín.- Máx.). **Promedio \pm desviación estándar. en la Tabla 2. Para facilitar la interpretación se presentan los antilogaritmos de los coeficientes de regresión: $(\beta): e^{\beta}$. Se constató en forma estadísticamente significativa que los niveles de vitamina D eran 25\% más bajos en hombres que en las mujeres, que por cada unidad de aumento del índice de Masa Corporal (IMC), estos disminuían en 4\% (Figura 3), y que el consumo de un suplemento de dicha vitamina los incrementaba en $56 \%$, en relación a los que no lo tomaban (Figura 4).

Se construyó un modelo multivariado con las variables que cruzaron el umbral de la significancia requerida. El modelo resultante (Tabla 3 ), era significativo $F(5,73)=6,82 ; p=0,000 ; R^{2}=0,32$. Tanto el IMC como el uso de un suplemento mantenían los efectos descritos en el análisis univariado. El género en este nivel de análisis no presentaba influencia significativa.

El uso de un suplemento de vitamina D producía una modificación del efecto del IMC (Figura 5), por lo que se sospechó la existencia de una interacción ${ }^{12}$ entre estas variables, pero esto se descartó con prueba de razón de verosimilitudes ${ }^{13}$ (Likelihood Ratio $\left.\chi^{2}(1)=1,56 ; p=0,212\right)$.

Los niveles de PTH de la muestra estuvieron dentro del rango normal $\left(49,9 \pm 17,2 \mathrm{pg} \cdot \mathrm{ml}^{-1}\right)$. Tampoco hubo diferencia significativa entre anestesiólogos $\left(49,6 \pm 18,2 \mathrm{pg} \cdot \mathrm{ml}^{-1}\right)$ y no anestesiólogos $\left(50,8 \pm 14,3 \mathrm{pg} \cdot \mathrm{ml}^{-1}\right)$.

Pese a existir una correlación inversamente proporcional significativa entre vitamina $\mathrm{D}$ y PTH (Figura 6), lo cual era esperable ${ }^{9}$, dicha asociación no se evidenció al analizar los niveles de PTH a través de las 3 categorías de vitamina D (Figura 7).

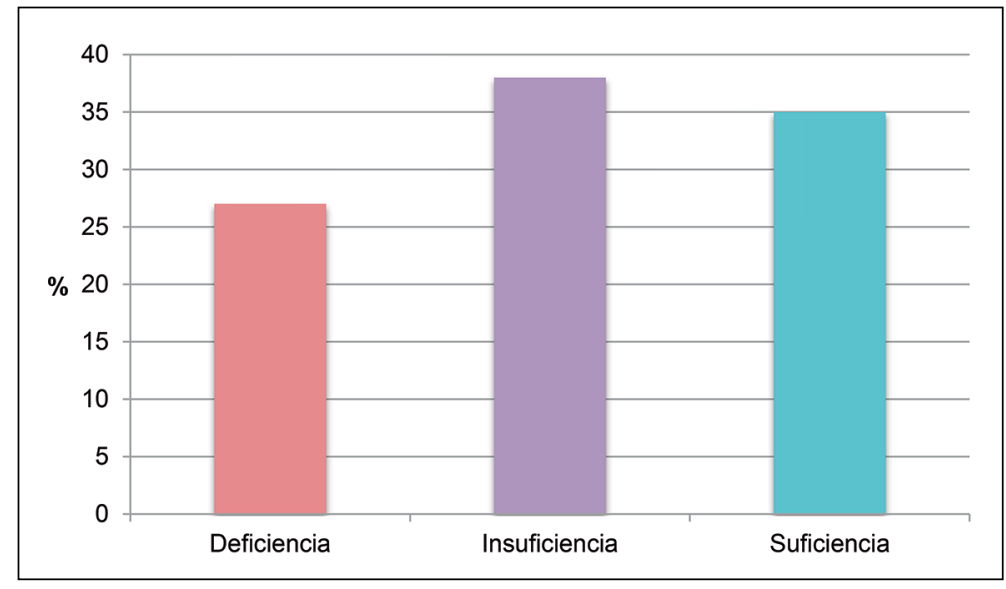

Figura 1. Niveles de vitamina $D$ en médicos. 


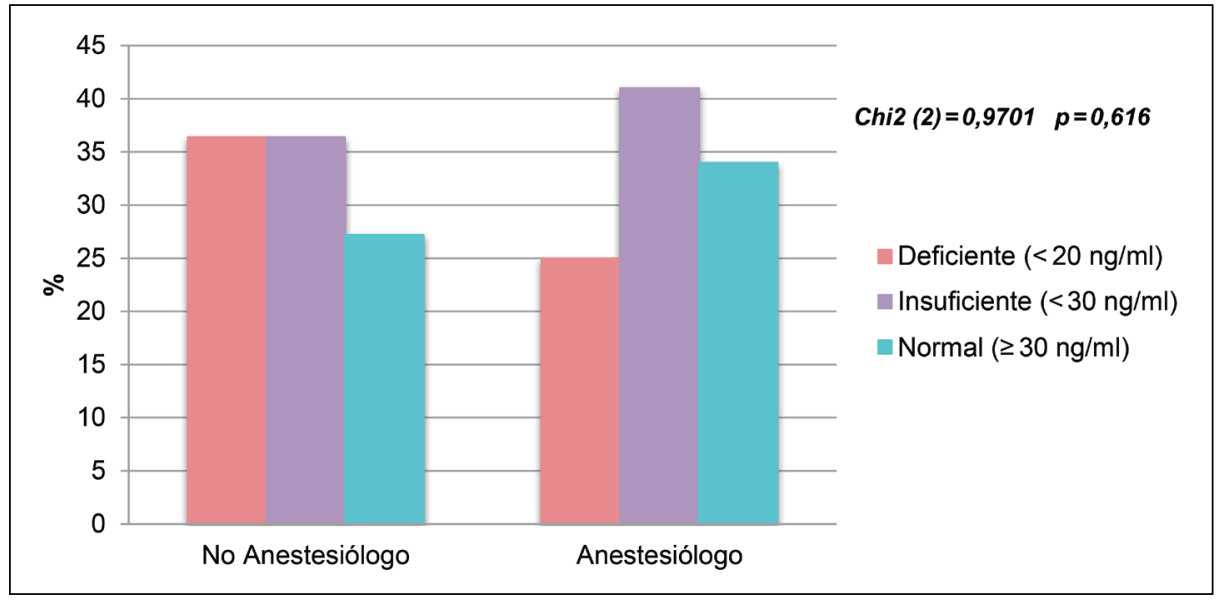

Figura 2. Estatus vitamínico según especialidad.

Tabla 2. Análisis univariado

\begin{tabular}{|c|c|c|c|c|c|}
\hline & Coeficiente () & $\mathbf{t}$ & \multicolumn{2}{|c|}{ I. Confianza 95\% } & p-valor \\
\hline Edad & 0,99 & $-0,90$ & 0,98 & 1,01 & 0,373 \\
\hline Género masculino & 0,75 & $-3,02$ & 0,62 & 0,91 & 0,003 \\
\hline $\mathrm{IMC}$ & 0,96 & $-2,69$ & 0,93 & 0,99 & 0,009 \\
\hline \multicolumn{6}{|l|}{ Fitzpatrick } \\
\hline 1 & Referencia & Referencia & \multicolumn{2}{|c|}{ Referencia } & Referencia \\
\hline 2 & 0,97 & $-0,23$ & 0,71 & 1,31 & 0,821 \\
\hline 3 & 1,07 & 0,50 & 0,81 & 1,43 & 0,622 \\
\hline 4 & 1,23 & 1,05 & 0,83 & 1,84 & 0,297 \\
\hline Anestesiólogos & 1,13 & 1,08 & 0,90 & 1,41 & 0,286 \\
\hline Horas semanales trabajo & 0,99 & $-1,69$ & 0,98 & 1,01 & 0,094 \\
\hline Actividad aire libre & 1,10 & 0,91 & 0,89 & 1,34 & 0,367 \\
\hline Uso de bloqueador solar & 1,20 & 1,75 & 0,96 & 1,48 & 0,083 \\
\hline Suplemento vitamina D & 1,56 & 4,32 & 1,27 & 1,92 & 0,000 \\
\hline Dieta rica en vitamina $D$ & 0,84 & $-1,53$ & 0,68 & 1,05 & 0,130 \\
\hline
\end{tabular}

Tabla 3. Análisis multivariado

\begin{tabular}{|lccccc|}
\hline & Coeficiente () & T & \multicolumn{2}{c|}{ I. Confianza 95\% } & p-valor \\
\hline Género masculino & 0,89 & $-1,12$ & 0,73 & 1,09 & 0,267 \\
IMC & 0,96 & $-2,28$ & 0,93 & 0,99 & 0,025 \\
Suplemento vitamina D & 1,56 & 4,47 & 1,28 & 1,90 & 0,000 \\
IMC*Suplemento* & 0,97 & $-1,20$ & 0,91 & 1,03 & 0,260 \\
\hline
\end{tabular}

*Interacción entre IMC y suplemento. 

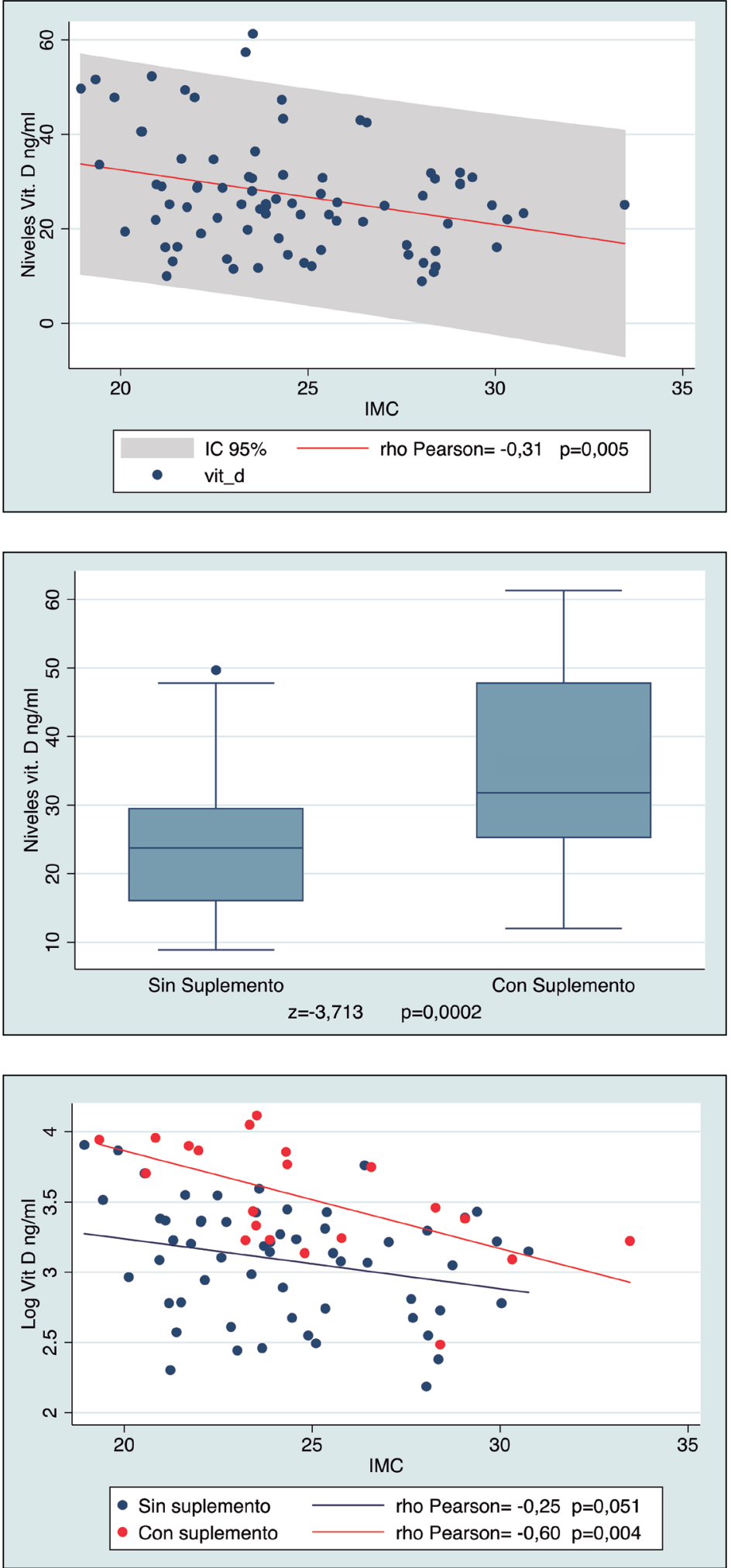

Figura 3. Correlación entre niveles de vitamina D e IMC.

Figura 4. Niveles de vitamina D según uso de suplemento.

Figura 5. Efecto del IMC sobre niveles de vitamina D según uso de suplemento. 

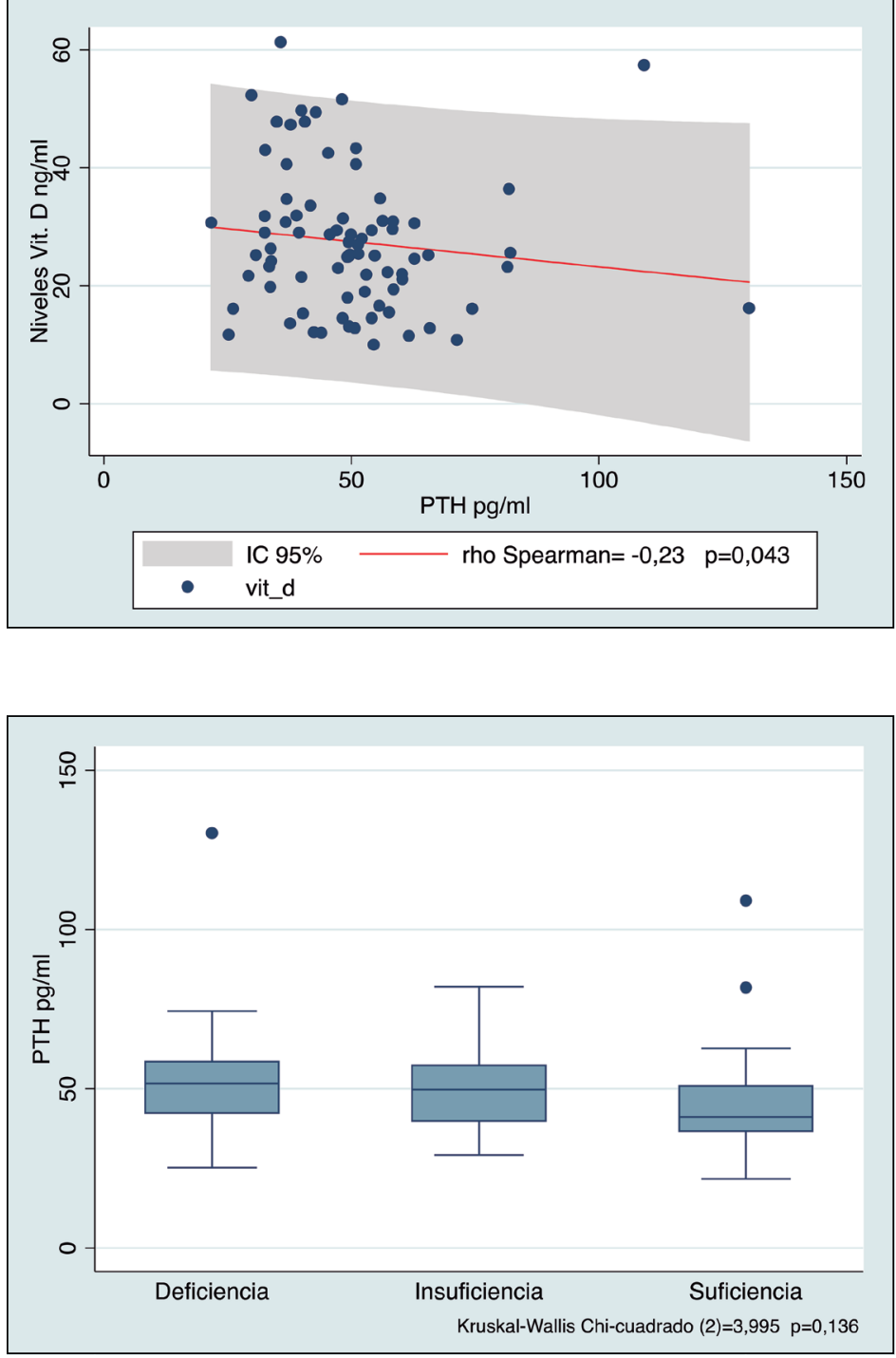

Figura 6. Correlación entre niveles de vitamina D y PTH.
Figura 7. PTH según categorías de vitamina D.

\section{Discusión}

Los resultados demostraron que en los médicos participantes de este estudio existía un déficit de vitamina $\mathrm{D}$ independientemente de la especialidad en que se desempeñaban. La proporción afectada en esta muestra fue incluso significativamente más alta que lo descrito en una reciente revisión sistemática ${ }^{6}$.

Fue destacable que esta deficiencia no estaba relacionada con aquellas variables que implicaran exposición al sol, incluida la coloración de la piel, horas de trabajo, deporte al aire libre y uso de blo- queador solar. Por otro lado, si se demostró una asociación inversa entre niveles de vitamina $\mathrm{D}$ e IMC. Esta relación ha sido previamente descrita ${ }^{14}$ pero el mecanismo causal no ha sido plenamente descubierto $^{14}$, se ha planteado la posibilidad de que exista secuestro de la vitamina en el tejido adiposo $^{15} \mathrm{o}$ bien un déficit asociado de la enzima 25 -hidroxilasa ${ }^{16}$. La única manera de soslayar esta deficiencia fue tomando un suplemento de vitamina $\mathrm{D}$. Hubo indicios de una interacción entre índice de masa corporal y suplemento que no pudo ser demostrada en esta muestra pero que sería importante continuar investigando. 
No se observó variación estacional de los niveles de vitamina $\mathrm{D}$, lo cual no tiene clara explicación y contradice los hallazgos de otro estudio chileno ${ }^{17}$ cuya unidad de análisis también fueron anestesiólogos. Los resultados de esta investigación se suman a la evidencia proporcionada por otras investigaciones de la literatura médica nacional ${ }^{18,19}$ que reportan una alta prevalencia de hipovitaminosis $\mathrm{D}$ en distintos grupos de la población chilena.

Pese a la existencia de una correlación inversamente proporcional entre niveles de vitamina $\mathrm{D}$ y $\mathrm{PTH}$, hubo 2 participantes pertenecientes a la categoría "suficiente" de vitamina D que exhibieron niveles elevados de PTH, en los cuales se sospechó un hiperparatiroidismo primario, de lo que fueron alertados.

Se podría haber mejorado la calidad de este estudio realizando las mediciones a fines del invierno o al menos en una misma estación del año. Puede ser discutible la inclusión de sujetos que estuvieran usando un suplemento de vitamina D pero uno de los fines de este estudio era exponer la realidad del problema en nuestra institución y por otro lado la ingesta de suplemento se incorporó como variable en el modelo de regresión multivariado lo que implica que se establecieron comparaciones entre sujetos equivalentes. Además, se podría haber optimizado la minuciosidad, tanto de la encuesta alimentaria como del registro del número de horas de exposición al sol y dosis del suplemento utilizado.

En conclusión, se demostró que la hipovitaminosis D afectaba por igual a los anestesiólogos y a los médicos que ejercían otras especialidades y la obesidad agravaba estos índices. El uso de un suplemento de vitamina $\mathrm{D}$ fue el único factor protector observado.

\section{Referencias}

1. Kennel KA, Drake MT, Hurley DL. Vitamin D deficiency in adults: When to test and how to treat. Mayo Clin Proc 2010; 85: 752-8.

2. Glendenning P. Measuring Vitamin D. Aust Presc 2015; 38: $12-5$.

3. Littlejohns TJ, Henley WE, lang IA, Annweiler C, Beauchet O, Chaves PHM, et al. Vitamin D and the risk of dementia and Alzheimer disease. Neurology 2014; 83: 920-8.
4. Murphy AB, Nyame Y, Martin IK, Catalona WJ, Hollowell CMP, Nadler RB. Vitamin D deficiency predicts prostate biopsy Outcomes. Clin Cancer Res 2014; 20: 2289-99.

5. Skerphedinsdottir SJ, Sigurdsson MI, Coursin DB, Head DE, Springman SR, Wang S, et al. Vitamin D deficiency in anesthesia department caregivers at the end of Winter. Acta Anaesth Scand 2014; 58: 802-6.

6. Sowah D, Fan X, Dennett L, Hagdvedt R, Straube S. Vitamin D levels and deficiency with different ocupations: a systematic review. BMC Public health 2017; 17: 519.

7. Aguilera G, Barberán M, Vargas J, Maldonado F, Brunet L, Caamaño E, et al. 25-hydroxyvitamin D deficiency among anaesthesiologists and Anaesthesia residents in Chile [letter to the editor]. Br J Anaesth 2017; 118: 9512.

8. Curiel-Lewandroski C. Risk factor for the Development of melanoma. En: Atkins MB, Tsao H, Corona R, editors. UpToDate; 2016. Disponible en: https://www. uptodate.com/contents/risk-factors-for-the-development-of-melanoma.

9. Holick MF. Vitamin D deficiency. N Engl J Med 2007; 357: 266-81.

10. American Board of Internal Medicine. ABIM Laboratory Test Reference Ranges [Actualizado enero 2019]. Disponible en: https://www.abim.org/ /media/ABIM\%20 Public/Files/pdf/exam/laboratory-reference-ranges.pdf.

11. Morgan SE, Reichert T, Harrison T. From numbers to words. Reporting statistical results for the social sciences. Kindle ed., Taylor \& Francis 2017.

12. Corraini P, Olsen M, Pedersen L, Dekkers OM, Vandenbroucke JP. Effect modification, interaction and mediation: an overview of theorethical insights for clinical investigation. Clin Epidemiol 2017; 9: 331-8.

13. Held L, Sabanés-Bové D. Frequentists properties of the Likelihood. En: Held L, Sabanés-Bové D, Editores, Applied Statistical Inference-Likelihood and Bayes: Springer-Verlag Berlin Heidelberg 2014 p: 106.

14. Saneei P, Salehi-Abargouei A, Esmaillzadeh A. Serum 25-hydroxy-vitamin D levels in relation to body mass index: a systematic review and meta-analysis. Obes rev 2013; 14: 393-404.

15. Pourshaidi LK. Vitamin D and obesity: current perspectives and future directions. Proc Nutr Soc 2014; 18-20 June, 1-10.

16. Roizen J, Long C, Casella A. “Obesity Decreases Hepatic 25-HydroxylaseActivity Causing Low Serum 25-Hydroxyvitamin D”. J Bone Miner Res 2019; 1-6.

17. Barberán M, Aguilera G, Brunet L, Maldonado F. Déficit de vitamina D. Revisión epidemiológica actual. Rev hosp Univ Chile 2014; 25: 127-34. 
18. González G, Alvarado JN, Rojas A, Navarrete C, Velásquez CG, Arteaga E. High prevalence of vitamin D deficiency in Chilean healthy postmenopausal women with normal sun exposure: additional evidence for a worldwide concern. Menopause 2007; 14 (3 Pt 1):
455-61.

19. Vallejo MS, Blümel JE, Lavín P, Torres C, Araos A, Sciaraffia C. Older women do not have seasonal variations of vitamin D levels. Menopause 2018; 25 (8): 912-7. doi:10.1097/gme.0000000000001103. 